\title{
Complete Coverage Path Planning and Obstacle Avoidance Strategy of the Robot
}

\author{
JunHui Wu, TongDi Qin \\ Institute of Modern Agricultural Science \& Engineering \\ Tongji University \\ 4800 Caoan Hwy, Jiading, Shanghai, P.R. China \\ E-mail: junhui_wu@163.com \\ qintongdi@yahoo.cn \\ Qiang Zhou \\ Shanghai Urban Green Engineering Co., Ltd \\ 1688 Jianchuan Hwy, Shanghai, P.R. China \\ E-mail: zhou.qiang@dushigreen.com \\ corresponding author
}

\begin{abstract}
In order to solve the problems of complete coverage path and obstacle avoidance with the mobile robot, the complete coverage planning was described first, and then the algorithm of the complete coverage path planning was analyzed. The complete traversal algorithm and the obstacle avoidance strategy of the robot around the barrier were put forward. Finally, the traversal control flow chart of the traversal robot implemented in Single Chip Microcomputer (SCM) was obtained. After the above analysis, the algorithm was simple, practical, and low repeatability, and high efficiency. The algorithms could effectively solve the difficulty of complete coverage path and obstacle avoidance with the robot.
\end{abstract}

Keywords - complete coverage path planning; obstacle avoidance strategy; the traversal robot; algorithm analysis; control flow chart

\section{INTRODUCTION}

The problems of path planning and obstacle avoidance have been a hot issue in the research of the intelligent mobile robot [1-5]. Obstacle avoidance is taking avoidance (to prevent collision) strategy when the robot moves along its working path. It is mainly aimed at the obstacle and the local environment of robot [6-7]. The complete coverage path planning algorithm of the mobile robot would be studied systematically, and then the obstacle avoidance of the complete coverage path planning algorithm would be explored.

\section{THE DESCRIPTION OF THE COMPLETE COVERAGE PLANNING}

\section{A. The Mathematical Description of the Traversal Planning}

Complete coverage path planning is a special path planning, set $\mathrm{S}=\{\mathrm{x}, \mathrm{y} \mid \mathrm{x}, \mathrm{y}$ is the point surrounded by a closed curve, that is, the entire range of the robot motion\}. Definition 1: reachable region $\mathrm{S}_{\Omega}=\{\mathrm{x}, \mathrm{y} \mid \operatorname{ocup}(\mathrm{x}, \mathrm{y})=0\} \subseteq \mathrm{S}$, and $\operatorname{ocup}(\mathrm{x}, \mathrm{y})$ is the possession function, the value is 0 when barrier-free, and is 1 when there is an obstacle; Definition 2:

\author{
Jie Chen, HuiPing Si, KaiYan Lin \\ Institute of Modern Agricultural Science \& Engineering \\ Tongji University \\ 4800 Caoan Hwy, Jiading, Shanghai, P.R. China \\ E-mail: chenjie18@yahoo.com.cn \\ sihuiping@tongji.edu.cn \\ ky.lin@163.com \\ ChiBin Zhang \\ Institute of Mechanical Engineering \\ Southeast University \\ 79 Suyuan Hwy, Jiangning, Nanjing, P.R. China \\ E-mail: chibinzhang@yahoo.com.cn
}

unreachable region $\mathrm{S}_{\Delta}=\{\mathrm{x}, \mathrm{y} \mid \operatorname{ocup}(\mathrm{x}, \mathrm{y})=1\} \subseteq \mathrm{S}$, and $\mathrm{S}_{\Omega} \cap \mathrm{S}_{\Delta}$ $=0$, and $\mathrm{S}_{\Omega} \cup \mathrm{S}_{\Delta}=\mathrm{S}$.

If the plane mobile robot is equivalent to a particle, then the traversal is a continuous path that the particle can continuously move and go through all the points in $\mathrm{S}_{\Omega}$. And the path is called feasible traversal trajectory, all of which are called feasible traversal trajectory cluster, which are expressed by the following formula:

$$
\mathrm{L}_{\Sigma}=\left\{l_{i}\left(x, y, x_{s}, y_{s}, x_{g}, y_{g}\right)\right\} \quad(i=1,2 \ldots n)
$$

Where $\left(x_{s}, y_{s}\right)$ and $\left(x_{g}, y_{g}\right)$ is the start and end coordinate values of the robot, and $(x, y),\left(x_{s}, y_{s}\right),\left(x_{g}, y_{g}\right) \subseteq S$.

\section{B. The Performance Indicators of the Complete Coverage Planning}

The common performance evaluation indicators of the complete coverage path planning of mobile robot are coverage area percentage and coverage overlapping rate.

$\mathrm{S}$ is the entire working space of the mobile robot, and $\mathrm{S}_{\Omega}$ is the reachable region, and $S_{h c}$ is the coverage area, and $S_{c c}$ is the iterate coverage area. 1) Coverage area percentage $J_{h c}$ refers to the percentage of the coverage area and the area of reachable region when the coverage of robot along feasible trajectory lines has already completed. 2) The coverage overlapping rate refers to the percentage of all the coverage overlapping area and reachable area.

$$
\begin{aligned}
& \mathrm{J}_{\mathrm{hc}}=\mathrm{S}_{\mathrm{hc}} / \mathrm{S}_{\Omega} \\
& \mathrm{J}_{\mathrm{cc}}=\mathrm{S}_{\mathrm{cc}} / \mathrm{S}_{\Omega} \\
& \mathrm{J}_{\mathrm{uc}}=1-\mathrm{J}_{\mathrm{hc}}
\end{aligned}
$$

There must be a certain degree of overlap among adjacent coverage region in order to avoid coverage blind spot, apparently, the smaller of overlap region the better. But due to the systematic errors, positioning error, the control accuracy and the environment of the robot, the overlapping area can not be too small. The uncovering area percentage $J_{u c}$ can be inferred from coverage overlapping rate, which refers to the percentage of the uncovering area and the area of reachable region when the traversal of the robot along 
feasible trajectory lines has already completed. The higher the performance of the robot, the higher the coverage area percentage; The lower the coverage overlapping rate, the better the coverage effect. The coverage overlapping rate and the uncovering area are combined to evaluate the complete coverage path planning.

\section{COMPLETE COVERAGE PATH PLANNING AROUND THE BARRIER BASED ON GRID MAPS}

The ideas of complete coverage path planning based around the barrier: (1) the parameters of the environmental boundary were obtained, according to the perceptual system, when the robot rounds along the environmental boundary. (2) The robot does the coverage task from the starting point, and first takes return traversal strategy based on the obstacle, then rounds obstacles to cover uncovering area, and finally reaches the purpose of covering all the area.

\section{A. Classification of Obstacles}

During the process of the entire path planning, the obstacles are divided into three categories, the first category is walls and obstacles which is close to the wall (on-wall obstacles). As long as the distance of obstacles and walls does not meet the minimum safety distance of the traversal robot, the robot can not pass, which is handled by on-wall obstacles. As these obstacles constitute the main boundary conditions (walls) and the additional boundary conditions (on-wall obstacles) of the robot work environment, which is classified as first class. The second category is the isolated obstacle farther away from the wall and smaller than the robot size, which is called the isolated obstacle. The third category is obstacles far away from the wall and larger than the robot size, which is called a general obstacle. Similarly, when the distance between two obstacles so close that the robot is unable to pass successfully, which is handled by an obstacle.

\section{B. The Border Learning of the Traversal Robot}

The border learning of the robot refers that the traversal robot automatically finds the position of the wall, and achieves the moving action along the walls and recording environmental parameters, such as environment size and onwall obstacles. Finally, the robot chooses a corner as a starting point to cover.

(1) The achievement of walking along the wall

The robot is to achieve the path planning with a certain regularity, and it is bound to select specific reference coordinates. The ideal reference is the wall in the room environment. The wall does not only have a good linear characteristic, while its distribution has a certain regularity. Therefore, during the coverage path planning, the walls of the room are selections as a basic reference for the entire two-dimensional spatial planning.

Then, the robot must further determine the positional relationship between its own and the reference. Initial position is close to the wall, and there is no obstacle between the robot and the wall. One direction is selected to complete the operation of the "along the wall". The environment parameters of the whole room are obtained, when the wall is as a reference.

The traversal robot must determine its starting point (temporary origin), after completing the action "along the wall".

For the setting of temporary origin, as long as it is located on the path of walking along the wall, and the traveling status of the robot has reached stability. As shown in Fig. 1, "---" is on behalf of the entire process of walking along the wall, and the starting point $\mathrm{A}$ is temporary origin.

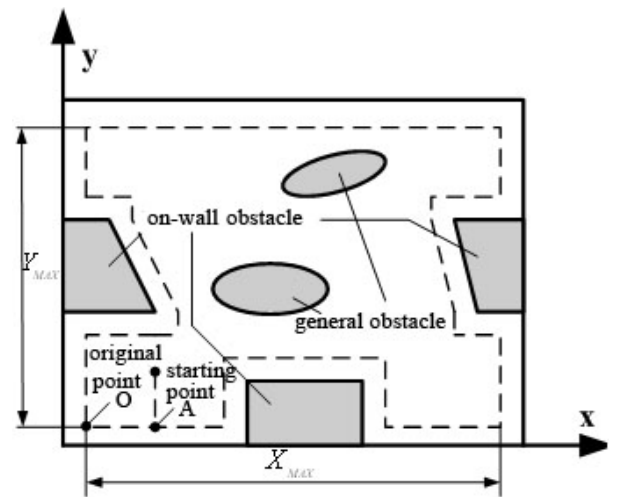

Figure 1. Border learning of the robot

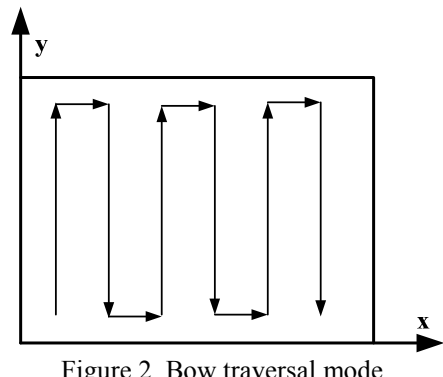

(2) Border learning

The traversal robot must first master the environmental situation in order to reasonably achieve the coverage path planning. After selecting the temporary origin $\mathrm{A}$, the traversal robot rounds the room a circle along the outer edge of the walls and on-wall obstacles, in a clockwise direction. The parameters of the environment are automatically recorded in accordance with the modeling method, and do the environmental modeling. The process of border learning is the process of actually creating the environment boundary map.

\section{Complete Coverage the Path Planning and Algorithm Analysis}

The bow type traversal mode is shown in Fig.2.

Base points refer to the special points used to characterize the obstacles in the environment (a grid cell in the grid method modeling). 


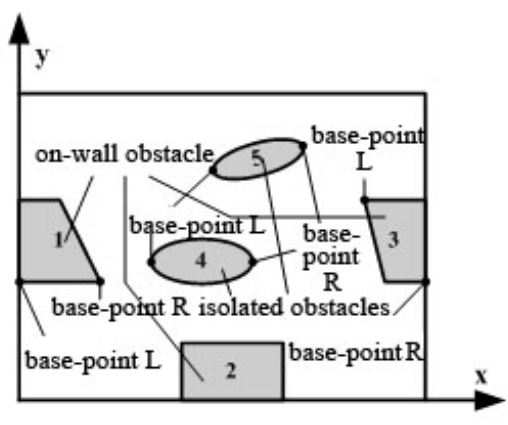

Figure 3. Classification of the environmental obstacles

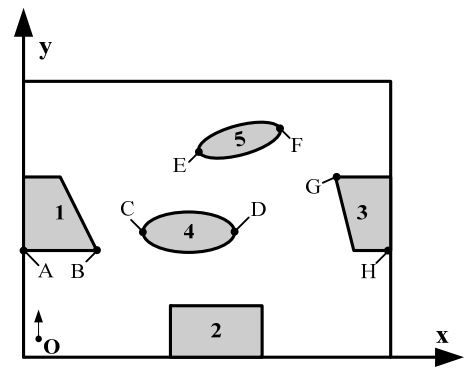

Figure 4. Environment layout of the traversal robot

It is not that each on-wall obstacle should select base points, and obstacle 2 does not need to select base points (as shown in Fig.3); For obstacle 1 and 3, base points R and L by the formula (4) and (5) are selected. For isolated obstacles and general obstacles, two base points $\mathrm{R}$ and $\mathrm{L}$ are selected by the formula (4) and (5). Selecting the base points of on-wall obstacles is finished in the process of border learning, however, selecting base points of the isolated obstacles is completed in the process of implementing traversal task.

For any one unit $\mathrm{C}(\mathrm{x}, \mathrm{y})$ of obstacles, $\mathrm{x}_{1} \leqslant \mathrm{x} \leqslant \mathrm{x}_{2}, \mathrm{y}_{1} \leqslant \mathrm{y}$ $\leqslant \mathrm{y}_{2}$,

$$
\begin{aligned}
L(x, y) & =\min \left\{y \mid C(x, y)_{x=x 1}\right\} \\
R(x, y) & =\min \left\{y \mid C(x, y)_{x=x 2}\right\}
\end{aligned}
$$

After the parameters of the environment borders and onwall obstacles are obtained, the robot selects the origin $\mathrm{O}$ and begins to perform the coverage path planning. The robot encounters the base point $\mathrm{L}$ of the obstacle, and moves $2 \mathrm{R}$ distance relative to the $\mathrm{X}$-axis direction, and traverses along the opposite direction of the original, that is, bow-type traversal. When the robot encounters the base point $\mathrm{R}$, it bypasses the obstacle.

Environmental layout is shown in Fig.4, the robot starts from the point $\mathrm{O}$, and traverses along the positive direction of the Y-axis. When the robot encounters obstacle 1, it moves $2 \mathrm{R}$ distance along the positive direction of $\mathrm{X}$-axis, and then continues to traverse along the negative direction of the Y-axis, until to the wall, and then moves $2 \mathrm{R}$ distance along the positive direction of the $\mathrm{X}$-axis. So until move to the right of the obstacle that point $\mathrm{B}$, according to the parameters of the border learning, this point is base point $R\left(x_{B}, y_{B}\right)$, then counterclockwise bypasses obstacles.
Subsequently, the robot continues to cover until it encounters the obstacle 4, according to the formula (4), selecting $\mathrm{C}$ as base point $L\left(x_{c}, y_{c}\right)$, and then continues to cover until it encounters point $\mathrm{D}$ suspected base point $\mathrm{R}$ of the obstacle 4, according to the formula (5) to determine whether the point $\mathrm{D}$ is base point $\mathrm{R}$ of obstacle 4 , dividing two cases discussed:

a) $\mathrm{D}$ is the base point $\mathrm{R}$ of the obstacle 4 , and the robot rounds along the obstacle 4 until the coordinates of the robot meets $x_{R} \leqslant x_{C}$; then the point is the starting position, and then covers to the positive direction of the $\mathrm{Y}$-axis.

b) $\mathrm{D}$ is not a base point of obstacle 4 , then the robot would find the real $\mathrm{R}$ base point $\mathrm{M}$ of obstacle 4 in the process of bypassing the obstacle 4 , then back to the suspected base point $\mathrm{D}$, to continue the original traversal until encounters the $\mathrm{R}$ base point $\mathrm{M}$, then covers according to the method of a).

The robot would be able to complete the coverage of the entire environment. Fig.5 is the map of coverage path planning of the traversal robot in the complex environments.

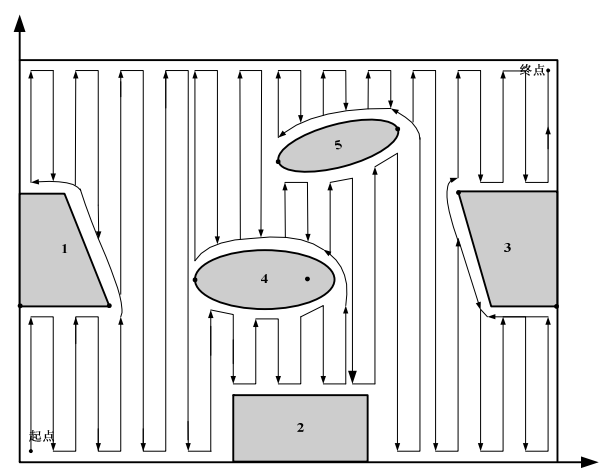

Figure 5. Complete coverage path planning

The experiments showed that the traversal algorithm was simple, high efficiency, but certain requirements were proposed simultaneously to the traversal robot:

a) The traversal algorithm required the robot to complete border learning, that is, rounding the wall. The traverse robot can walk a straight line and make a rotation.

b) The traversal algorithm required that the robot perceptual system can perceive environment walls, corners and faults.

\section{THE OBSTACLE AVOIDANCE STRATEGY OF ROBOT COMPLETE TRAVERSE PATH PLANNING}

The design of obstacle avoidance strategy primarily resolves how the robot avoids obstacles optimally. The problem forms a variety of methods: behavior learning method based on the basic action [8], block gradually approaching of dividing flat into multiple pixel [9], Voronoi graphical method [10], and so on. Traversal robots researched in this subject are robots indoors executing traversal movement, according to their tasks, the obstacle avoidance strategies are to consider the following questions:

a) Work security. Certainly there are obstacles in the indoor environment (such as walls, furnitures, etc.), taking 
into account the security issues of the robot, when the robot closes and encounters obstacles, the robot must stop the movement.

b) Traversal integrity. The obstacle avoidance of traditional mobile robots is called immediately the obstacles avoidance procedures after discovering the obstacles. The traversal robot as much as possible up to the coverage area, therefore, traversal robot should sufficiently close to obstacles, but also to avoid the collision, that is, the robot can not be far to avoid obstacles, but should be close to the obstacles to bypass.

\section{A. The Obstacle Avoidance Strategy Based on the Adjacent Multi-sensor}

During the movement of the robot, the information of the adjacent ultrasonic and infrared sensor was fused, and the corresponding type of reaction was derived by the fusion method of production rules [11]. At last, the obstacle avoidance method of the robot was got.

In operation of the robot, the distribution information based on the obstacle which is obtained according to the ultrasonic sensor in each direction is the steering conditions of the robot, which is the premise of the production rule base. The conclusion of the production rule base is that the robot takes which obstacle avoidance strategy or obstruction type. The production rule base is structured by combining these premises and conclusions, as shown in Table 1.

TABLE 1. THE PRODUCTION RULE BASE OF THE TRAVERSAL ROBOT

\begin{tabular}{|c|c|c|c|c|c|c|c|c|}
\hline Rules & U1 & $\mathrm{U} 2$ & U3 & $\mathrm{U} 4$ & U5 & U6 & U7 & Conclusion \\
\hline 1 & 0 & 0 & 0 & 0 & 0 & 0 & 0 & $\begin{array}{l}\text { There is no obstacle in front and on the right and on the left. The } \\
\text { robot does the linear motion. }\end{array}$ \\
\hline 2 & 0 & 0 & 0 & 0 & 0 & 1 & 1 & $\begin{array}{l}\text { There is no obstacle in front and on the left, and there is an obstacle } \\
\text { on the right. The robot does the linear motion along the edge of the right } \\
\text { obstacle. }\end{array}$ \\
\hline 3 & 1 & 1 & 0 & 0 & 0 & 0 & 0 & $\begin{array}{l}\text { There is no obstacle in front and on the right, and there is an obstacle } \\
\text { on the left. The robot does the linear motion along the edge of the left. }\end{array}$ \\
\hline 4 & 0 & 0 & 0 & 1 & 0 & 0 & 0 & $\begin{array}{l}\text { There is an obstacle in front and smaller than the robot. The robot } \\
\text { does the isolation-style obstacle avoidance motion. }\end{array}$ \\
\hline 5 & 0 & 0 & 1 & 1 & 1 & 0 & 0 & $\begin{array}{l}\text { There is an obstacle in front and larger than the robot. The robot } \\
\text { does the retrievable obstacle avoidance movement }\end{array}$ \\
\hline 6 & 0 & 1 & 1 & 0 & 0 & 0 & 0 & $\begin{array}{l}\text { There is an obstacle toward the left front and larger than the robot. } \\
\text { The robot does the rounding barrier-type obstacle avoidance movement } \\
\text { for general obstacles. }\end{array}$ \\
\hline 7 & 0 & 0 & 0 & 0 & 1 & 1 & 0 & $\begin{array}{l}\text { There is an obstacle toward the right front and larger than the robot. } \\
\text { The robot does the retrievable obstacle avoidance movement }\end{array}$ \\
\hline 8 & 0 & 0 & 1 & 1 & 1 & 1 & 1 & obstacle avoidance movement of concave wall \\
\hline 9 & 0 & 0 & 0 & 0 & 0 & 0 & 1 & obstacle avoidance movement of convex wall \\
\hline
\end{tabular}

Where "1" represents the feedback signal of the ultrasonic sensor, and "0" represents that there was no feedback signal with the ultrasonic sensor, or feedback signal exceeded the set value. The rules of table 1 are suit for the robot learning in the counterclockwise along the border and traversal movement.

The discriminant process of the production system is shown in Fig.6, the robot constantly matches the premise of the rule in the rule base with the data in the current database during the running. When the match is successful, the conclusion of acquiring the matching rules will be the next step action of the robot; or else, continuing to match the next rule with the data in the current database, until uses up all the rules.

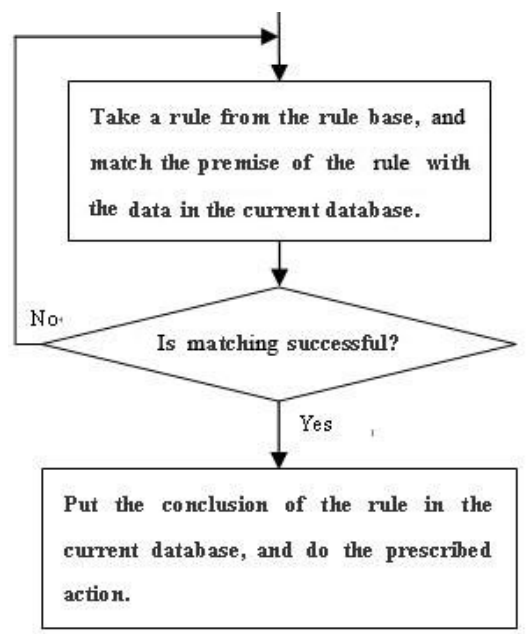

Figure 6. Discriminant flow of the production system

\section{B. The Type Analysis of the ObstacleAvoidance With the} Traversal Robot

a) The obstacle avoidance of convex walls

The question of convex wall is that the signal of one detection direction suddenly disappears during the robot in the process of border learning. For the obstacle avoidance 
behavior of the convex wall is shown in Fig.7, the robot does the linear motion along the left wall in position 1 , and then to position 2 the left wall signal disappears, according to the production rules, the robot performs the obstacle avoidance of convex wall. The robot simply needs revolver as a fixed wheel, the right rotation as a jog wheel, and turns $90^{\circ}$, and then the robot can do the rectilinear movement along the new wall.

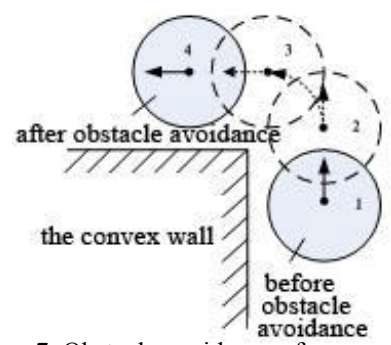

Figure 7. Obstacle avoidance of convex walls

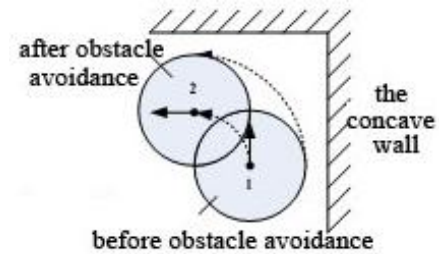

Figure 8. Obstacle avoidance of concave walls

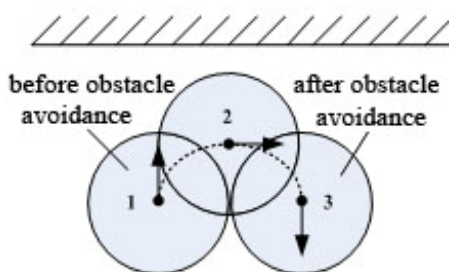

Figure 9. Retrievable obstacle avoidance

b) The obstacle avoidance of concave walls

A most typical situation of appearing concave walls is that the robot encounters a corner, as shown in Fig.8. The robot in position 1 obtains the obstacle information in front and on the right through the sensor, and the robot takes the obstacle avoidance of concave wall according to the production rules. The robot needs revolver as the center of rotation, and turns $90^{\circ}$, reaching position 2, then continues to do the movement along the wall.

c) Return-style obstacle avoidance

Return-style obstacle avoidance should meet two conditions: First, the robot runs the opposite direction with the original after obstacle avoidance; second, the run route of robot before and after the obstacle avoidance should be two parallel lines, and 2R distance away. As shown in Fig.9, when the robot runs to position 1 , there is an obstacle ahead, according to the production rules, the implementation of the return type of obstacle avoidance is obtained. The robot needs the right wheel as the center of rotation, and turns $180^{\circ}$, reaching the position 3, and then continues for reciprocating motion of the bow-shaped.

d) Obstacle avoidance method of the isolated obstacle
The way of rounding the obstacles is taken when the robot encounters the isolated obstacle. The entire process of obstacle avoidance is achieved by two ways of revolving and straight-line walking. As shown in Fig.10, the robot in position 1 determines that there is an isolated-obstacle in front, and executes as follows: counterclockwise revolving $90^{\circ} \rightarrow$ clockwise revolving $90^{\circ} \rightarrow$ straight line walking $\rightarrow$ clockwise revolving $90^{\circ} \rightarrow$ counterclockwise revolving $90^{\circ}$, and completes obstacle avoidance method of isolation-obstacles.

e) Obstacle_avoidance of general obstacles

Specific obstacle avoidance process is shown in Figure 11 , the robot uses the return type of obstacle avoidance running from position 1 to position 3 , then by now the robot does not detect the obstacle in front, then counterclockwise revolving $90^{\circ} \rightarrow$ walk straight $\rightarrow$ clockwise revolving $90^{\circ}$, by now the robot returns to the same line with the position 1 , and the robot continues to traverse.

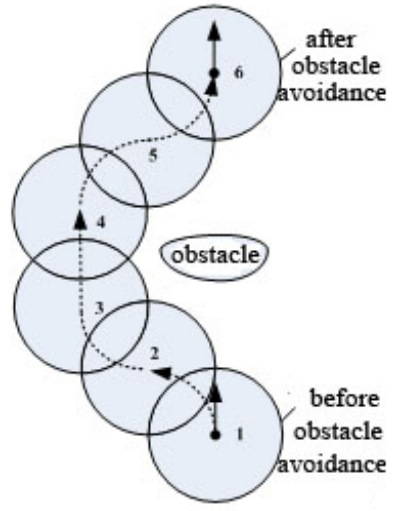

Figure 10. Obstacle avoidance of isolated obstacles

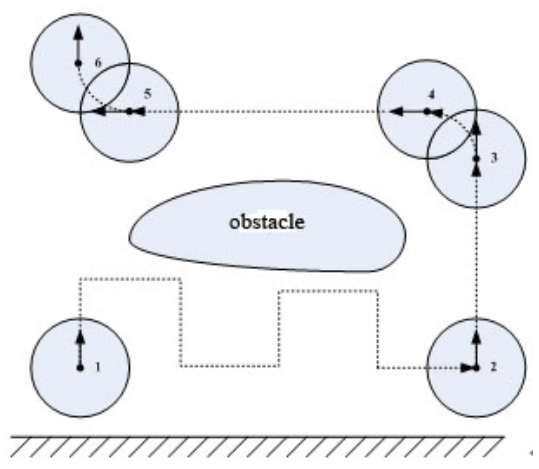

Figure 11. Obstacle avoidance of general obstacles

\section{THE CONTROL PROGRAM FLOW OF OBSTACLE AVOIDANCE WITH TRAVERSAL ROBOT}

The following discussion is that the path planning and obstacle avoidance strategy mentioned above how to achieve on the Single Chip Microcomputer (SCM). The control flow chart of traversal robot is shown in Fig. 12. After the control system of traversal robot starts, the robot begins to do the voluntary movement. First the robot 
determines whether in the wall position, if not, then automatically walks along a straight line to the location of the wall, and then counterclockwise rounds the environment circle, finally stops at the corner near to the initial position, which is the starting point of the robot work. The robot proceeds from the starting point, and the bow traversal is done, also the environment parameters are obtained by the perceptual system, to create an electronic map. The estimate of production rules for the information is done to complete the obstacle avoidance.

The obstacle avoidance control flow of the traversal robot is shown in Fig.13. Robot detects the area in front during the process of traversing. If there is no obstacle in the set distance, the robot continues the original action; or else, the robot has to determine the type of obstacles. If the obstacle is isolated-obstacle, the robot takes obstacle avoidance of isolated obstacle; If the obstacle is general obstacles, the robot takes obstacle avoidance of the general obstacles.
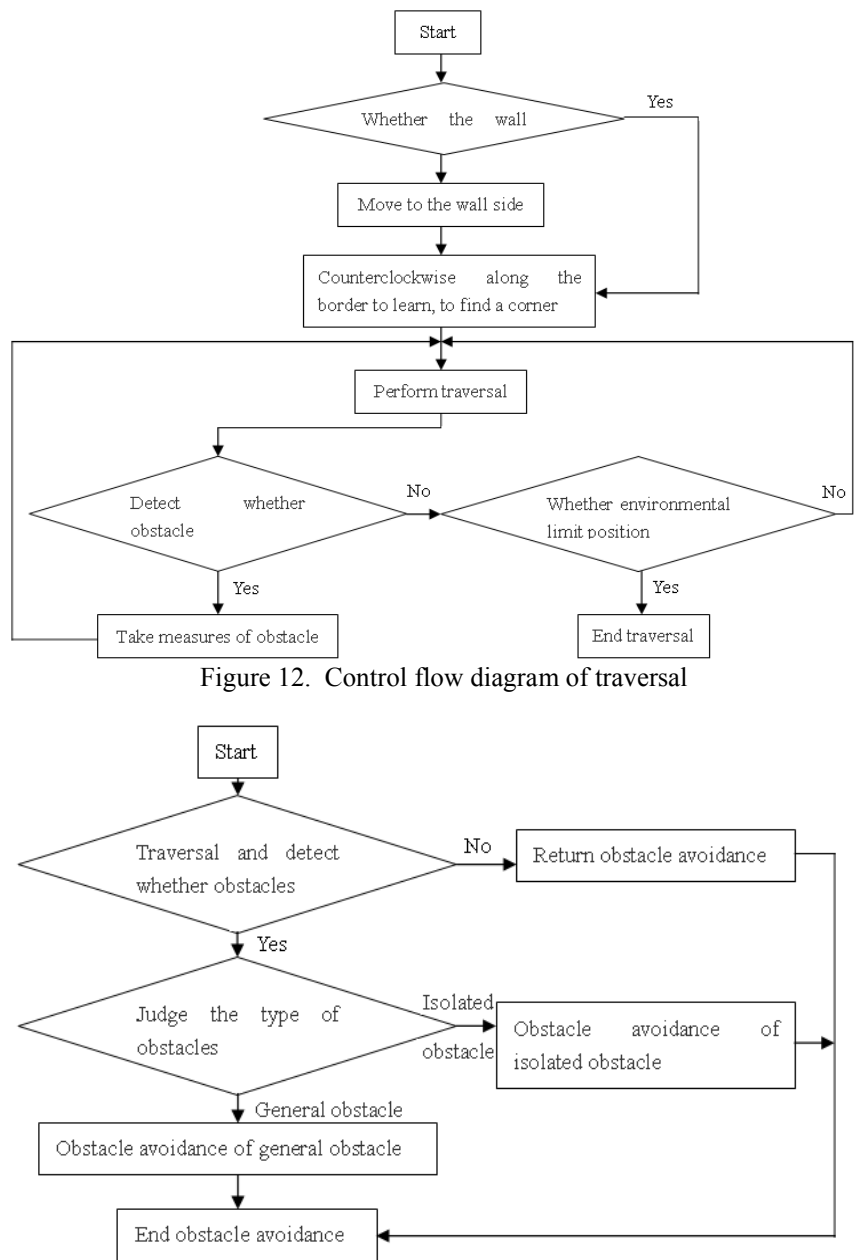

Figure 13. Control flow diagram of obstacle avoidance

\section{SUMMARY}

This paper analyzed the algorithm of complete coverage path planning, and the complete coverage algorithm of robot and obstacle avoidance strategy were put forward around the barrier. After the analysis, the algorithm is simple, practical, and low repeatability, and high efficiency.

\section{ACKNOWLEDGMENT}

This paper was sponsored by a key scientific research project of Shanghai (Project No. 11DZ2211104) and National Engineering Research Center of Protected Agriculture (Project No. 2011BAD43B01 and Project No. 2011BAD43B02).

\section{REFERENCES:}

[1] E.Prassler, A..Pitter, C.Schaeffer, P.Fiorini. A short history of cleaning robots. Autonomous Robots, vol.3, pp. 211-226, 2000.

[2] F.Yasutomi, D.Takaoka, M.Yamada, K.Tsukamoto. Cleaning robot control. In Proc. of IEEE Intl. Conf.on Robotics and Automation, Philadelphia, USA, 1988, pp. 1839-1841.

[3] R.D.Schraft, U.Brauning, T.Orlowski, M.Hornemann. Automated cleaning of windows on standard facades .Automationin Construction, vol.5-6, pp. 489-501, 2000.

[4] K.Kamiya, S.Iida, T.Koyama, et al. Vehicle control for clean room mobile robot. In Proc.Of Intl.Robots and Vision Con.Detroit, USA, 1997, pp. 1-15.

[5] D.C.MacKenzie, T.R.Baelh. Making a clean sweep : Behavior based vacuuming. In Proc.of AAAI Fall Symp.on Instantiating Real-World Agents, Raleigh, USA, pp. 93-98, 1993.

[6] A.Meystel,Autonomous Mobile Robots Vehicles with Cognitive Control,World Scientific Publishing Co. Pte. Ltd. 1991.

[7] T.A.Linden, J.P.Marsh and D.L.Dove. Architecture and early experience with planning for the ALV, Proc.IEEE Int. Conf.on Robotices and Automation, Vol.3, San Francisco, 1986.

[8] Koren Ward and Alexander Zelinsky. Acquiring Mobile Robots Behaviors by Learning Trajectory Velocities with Multiple FAM Matrices, Proceeding of the IEEE International Conference on Robotics \& Automation, Leuven, Belgium, May 1998.

[9] Cao, Z.L., Huang, Y., Hall, E.L. Region Filling Operations with Random Obstacle Avoidance for Mobile Robots. Journal of Robotic Systems, vol.2, 1988, pp. 87-102.

[10] Keiji Nagatani, Howie Choset, Sebastian Thrum. Towards Exact Localization without Explicit Localization with Generalized Voronoi Graph, Proceeding of the IEEE International Conference Robotics \& Automation Leauven, Belgium, May 1998.

[11] Y.B. Jiang. The vacuuming robot system development and obstacle avoidance problems. Beijing: Mechanical and Electronic Engineering, University of Science and Technology Beijing, 2005. 